\title{
Output regulation using new sliding surface with an implementation on inverted pendulum system
}

\author{
Asif Chalanga ${ }^{\mathrm{a}}$, Machhindranath Patil ${ }^{\mathrm{b}}$, Bijnan Bandyopadhyay $^{\mathrm{c}, *}$, Hemendra Arya $^{1}$ \\ ${ }^{a}$ Department of Electronic and Electrical Engineering, University College London, London WC1E 6BT, U.K. \\ ${ }^{b}$ Department of Instrumentation Engineering, V.E.S. Institute of Technology, Mumbai, India. \\ ${ }^{c}$ IDP in Systems and Control Engineering, Indian Institute of Technology Bombay, India. \\ ${ }^{d}$ Department of Aerospace Engineering, Indian Institute of Technology Bombay, India.
}

\begin{abstract}
In this paper constant reference output tracking is achieved using second order sliding mode (SOSM) control. To achieve tracking a new sliding surface is proposed and to ensure sliding motion super-twisting control (STC) is employed. The proposed sliding surface is more general, and it can be used for constant reference tracking in both minimum phase and non-minimum phase systems. The proposed method is validated on the unstable non-minimum phase inverted pendulum system and also the results are compared with the first order sliding mode control (SMC) in simulation and experimentally both.
\end{abstract}

Keywords: Non-minimum phase system, output regulation, second order sliding mode, super-twisting control.

\section{Introduction}

Output regulation is one of the classical control problems and for the class of non-minimum phase systems the problem becomes more difficult (Isidori, 2013; Byrnes and Isidori, 2000). In earlier work, Francis and Wonham (1976) introduced internal model principle to solve the tracking problem for linear systems by incorporating the dynamical model of the plant in an exosystem that generates the reference signal. Further, this work is generalized by Isidori and Byrnes (1990) by identifying the acceptable dynamics on a particular center manifold.

Sliding mode control (SMC) has been a topic of great interest to researchers since it's inception as it offers simplicity in control structure, robustness and insensitivity to a class of disturbances (see Utkin et al., 2009; Shtessel et al., 2014; Edwards and Spurgeon, 1998; Boiko, 2008; Kamal et al., 2014; Hung et al., 1993; Utkin, 1993).

\footnotetext{
${ }^{*}$ Corresponding author.

Email addresses: a.chalanga@ucl .ac.uk (Asif Chalanga), machhindra.patil@ves.ac. in (Machhindranath Patil),

bjnan@sc.iitb.ac.in (Bijnan Bandyopadhyay),

arya@aero.iitb.ac.in (Hemendra Arya)
}

Preprint submitted to European Journal of Control
Numerous work on SMC in the direction of output regulation has been found in literature such as a work by Edwards and Spurgeon (1996) addresses observer based design, high performance control using nonlinear sliding surface by Bandyopadhyay and Fulwani (2009). In recent work by Bandyopadhyay and patil, reduced order SMC design method for continuoustime systems has been addressed in (Bandyopadhyay and Patil, 2015) and for discrete-time system in Patil and Bandyopadhyay (2013a).

Numerous literature found on the control of electro-mechanical systems via the first or higher order SMC. For instance, see Yan et al. (2000); Utkin and Chang (2002); Chalanga et al. (2015); Chalanga and Plestan (2017); Khimani and Rokade (2017). However, in most of the cases, first order SMC may not be acceptable for the discontinuous nature of the control as it leads to chattering. Chattering may excite unmodeled dynamics, which may hinder the stability of the system or may lead to actuator failure. To make the first order SMC continuous, saturation function can be employed instead of switching function. However, use of saturation function loses the robustness properties. 
Many authors came with different design procedures that eliminate the chattering effect viz. (Bartolini et al., 1998; Levant, 2010). Using higher order sliding mode (HOSM), it is possible to suppress the chattering effect without losing the robustness property (Levant, 1993, 1998, 2003). Therefore in this article, super-twisting control (STC), which is one of second order sliding mode controls, has been employed. This ensures the finite time reachability of the trajectory on the proposed sliding surface. For more on STC design, refer Behera et al. (2017); Polyakov and Poznyak (2009); Moreno and Osorio (2012); Davila et al. (2005).

Often output tracking in SMC framework is achieved via stabilization of the error between the system and the desired state trajectory, while the desired state trajectory is generated using exosystem. In this article, an effort is made to propose a method for output tracking that involves a novel switching surface and second order sliding mode controller without an exosystem. This paper essentially contributes,

i. A design of novel sliding surface that involves the system states and a reference signal. A sliding surface is designed for the stable sliding motion while inclusion of the reference signal ensures the tracking during sliding motion. A feed-forward gain, which is necessary for sliding surface design to eliminate the asymptotic tracking error during sliding motion is computed.

ii. A design of super-twisting control that initiates the sliding motion on the proposed surface in finite time.

iii. To validate the proposed method experimentally, the sliding surface and the controller as stated in (i) and (ii) are designed and implemented on inverted pendulum laboratory setup developed by educational control products (ECP, 2004). The experimental results obtained are compared with simulation results and they found to be quite satisfactory.

The paper is organized as follows. Section 2 discusses motivation behind the work. Section 3 details the main results of the paper. An application of the proposed method to the inverted pendulum system is discussed in Section 4. Section 5 contains simulation and experiments results followed by the conclusion.

\section{Motivation}

Consider the linear time invariant (LTI) system,

$$
\begin{aligned}
& \dot{x}=A x+B(u+d) \\
& y=C x,
\end{aligned}
$$

where $x \in \mathbf{R}^{n}, u \in \mathbf{R}$ and $y \in \mathbf{R}$ are the states, input and output of the system, respectively. $d \in \mathbf{R}$ represents the matched disturbance. Assume-

1. $(A, B)$ is controllable.

2. $(A, C)$ is detectable and $(A, B, C)$ has no zero at $s=0$.

First assumption is made for existence of the control law while the second assumption ensures the exact asymptotic tracking of the desired step reference.

If we consider $d=0$, then for such a system asymptotic tracking of constant reference can be achieved by the control law,

$$
u=-K x+\bar{G} r
$$

where $K$ is chosen so that $(A-B K)$ is Hurwitz, $r$ be $0,0.5,0$ the step signal and $\bar{G}$ be the constant that can be computed as,

$$
\bar{G}=-\left[C(A-B K)^{-1} B\right]^{-1} .
$$

This result is well known in the literature (see Franklin et al., 1994; Chen et al., 2003). However, controller (2) may not perform well in the presence of disturbance $d$. Therefore, this problem can be formulated in the SMC frame-work that completely rejects the disturbance. SMC based output tracking controller is usually designed for the stabilization of the error states. Therefore, it is necessary to transform the system in terms error variables that represent error between system states and desired states generated by exosystem. Recently in (Patil et al., 2018), a method for the non-causal output tracking of constant as well as time-varying reference via reduced order sliding mode control with an application to the inverted pendulum system (ECP, 
2004) has been proposed. However, in (Patil et al., 2018), the reduced order controller was designed for the stabilization of the error between the system trajectory and desired state trajectory. Also, the reduced order design ensures the asymptotic stability of the error locally in the predefined domain.

This motivates us to design a SMC controller for output tracking of constant reference signal that is simple, robust against the matched disturbance and does not require to transform the system in error coordinates.

\section{Main results}

Let there exist a linear transformation $Z=T x$ that transforms the system (1) into the regular form due to Lukyanov and Utkin (1981),

$$
\begin{aligned}
& \dot{Z}=A_{\text {reg }} Z+B_{\text {reg }}(u+d), \\
& y=C Z .
\end{aligned}
$$

where

$$
\begin{aligned}
A_{\text {reg }} & =\left[\begin{array}{ll}
A_{11} & A_{12} \\
A_{21} & A_{22}
\end{array}\right] ; Z=\left[\begin{array}{l}
Z_{1} \\
Z_{2}
\end{array}\right] \\
B_{\text {reg }} & =\left[\begin{array}{l}
B_{1} \\
B_{2}
\end{array}\right]=\left[\begin{array}{c}
0_{(n-1) \times 1} \\
1
\end{array}\right] \\
C & =\left[\begin{array}{ll}
C_{1} & C_{2}
\end{array}\right] .
\end{aligned}
$$

Therefore, a system (4) can be partitioned as,

$$
\begin{aligned}
& \dot{Z}_{1}=A_{11} Z_{1}+A_{12} Z_{2} \\
& \dot{Z}_{2}=A_{21} Z_{1}+A_{22} Z_{2}+(u+d) \\
& y=C_{1} Z_{1}+C_{2} Z_{2}
\end{aligned}
$$

where $Z_{1} \in \mathbf{R}^{n-1}$ and $Z_{2} \in \mathbf{R}$. As the system is represented in regular form, problem of output tracking can be solved by introducing a novel sliding surface.

\subsection{Sliding surface}

An ideal sliding mode is said to take place on a manifold

$$
\mathbf{S}=\left\{Z \in \mathbb{R}^{n} \mid \sigma(Z)=0\right\}
$$

if the trajectory $Z(t)$ reaches the manifold $\sigma(t)=0$ in finite time $t_{r} \in \mathbb{R}^{+}$and remained thereon for all $t \geq t_{r}$. Also, during sliding mode $\|Z\| \rightarrow 0$ as $t \rightarrow \infty$. However, this implies, $\|y\| \rightarrow 0$ as $t \rightarrow \infty$. Therefore, in order to achieve output tracking, the novel sliding surface is introduced in the following theorem.

Remark 1. In sliding mode frame-work, often for the output tracking, the system in regular form such as one in (5) is required to be transformed into error variables that essentially are error between system and desired state trajectory and; then the sliding surface is designed for the robust asymptotically stable motion of the error variables, for instance see work by Patil and Bandyopadhyay (2013b). However, in this article, proposed design of the sliding surface and the control does not require reference trajectory

Theorem 1 (Novel sliding surface). Suppose that $r$ is a constant tracking signal. Let trajectory $Z(t)$ of the system (4) be restricted on the switching surface,

$$
\sigma:=F^{\top} Z-G r=: F_{1} Z_{1}+Z_{2}-G r=0
$$

then output $y$ tracks the reference input $r$ asymptotically if

$$
G=-\left[\left(C_{1}-C_{2} F_{1}\right) A_{c l}^{-1} A_{12}-C_{2}\right]^{-1}
$$

and $F_{1}$ is chosen such that $A_{c l}:=\left(A_{11}-A_{12} F_{1}\right)$ is Hurwitz.

Proof. During sliding motion, $\sigma=0$. So from (7),

$$
Z_{2}=-F_{1} Z_{1}+G r
$$

Substitute in (5a),

$$
\begin{aligned}
\dot{Z}_{1} & =\left(A_{11}-A_{12} F_{1}\right) Z_{1}+A_{12} G r \\
& =A_{c l} Z_{1}+A_{12} G r \\
\Rightarrow Z_{1}(t) & =\exp \left(A_{c l} t\right) Z_{1}(0)+\int_{0}^{t} \exp \left(A_{c l}(t-\tau)\right) A_{12} G r d \tau \\
& =\exp \left(A_{c l} t\right) Z_{1}(0)+A_{c l}^{-1}\left(-I+\exp \left(A_{c l} t\right)\right) A_{12} G r
\end{aligned}
$$

As $A_{c l}$ is stable by design, therefore

$$
Z_{1}(\infty)=-A_{c l}^{-1} A_{12} G r
$$


Also, from (9)

$$
\begin{aligned}
Z_{2}(\infty) & =-F_{1} Z_{1}(\infty)+G r \\
& =\left(F_{1} A_{c l}^{-1} A_{12}+1\right) G r
\end{aligned}
$$

Thus

$$
\begin{aligned}
y(\infty) & =C_{1} Z_{1}(\infty)+C_{2} Z_{2}(\infty) \\
& =-C_{1} A_{c l}^{-1} A_{12} G r+C_{2} F_{1} A_{c l}^{-1} A_{12} G r+C_{2} G r \\
& =\left(\left(-C_{1}+C_{2} F_{1}\right) A_{c l}^{-1} A_{12}+C_{2}\right) G r
\end{aligned}
$$

Clearly, If we choose

$$
G=-\left(\left(C_{1}-C_{2} F_{1}\right) A_{c l}^{-1} A_{12}-C_{2}\right)^{-1}
$$

this gives $y(\infty)=r$. Hence proved

\subsection{Sliding mode control}

It is well known that sliding mode control (SMC) is twostep design procedure, first step is to design a sliding surface and second step is controller design that initiates the sliding mode in finite time. Therefore, it is necessary to design a control that enforces the sliding motion along the surface (7).

\subsubsection{First order SMC}

For a system (4), typically a first order sliding mode can be given by,

$$
u=-\left(F^{\top} B_{\mathrm{reg}}\right)^{-1}\left(F^{\top} A_{\mathrm{reg}} Z+u_{\mathrm{FSMC}}\right),
$$

where $u_{\mathrm{FSMC}}$ is the first order SMC, which is defined as

$$
u_{\mathrm{FSMC}}=M \operatorname{sign}(\sigma),
$$

In first order SMC, it is assumed that disturbance is bounded. To show the finite time reaching of the trajectory on the sliding surface, consider the Lyapunov function $V=\frac{1}{2} \sigma^{2}$. So the time derivative of the Lyapunov function along the trajectory of (4) is given by,

$$
\begin{aligned}
\dot{V} & =\sigma \dot{\sigma} \\
& =\sigma\left(F^{\top} \dot{z}\right) \\
& =\sigma\left(F^{\top}\left(A_{\mathrm{reg}} z+B_{\mathrm{reg}}(u+d)\right)\right. \\
& =\sigma\left(-M \operatorname{sign}(\sigma)+F^{\top} B_{\mathrm{reg}} d\right) \\
& \leq-|\sigma|\left(M-F^{\top} B_{\mathrm{reg}} d \operatorname{sign}(\sigma)\right) \\
& =-(2 V)^{\frac{1}{2}}\left(M-F^{\top} B_{\mathrm{reg}} d \operatorname{sign}(\sigma)\right)
\end{aligned}
$$

Let the disturbance bound be $|d(t)|<\delta$. So if we choose the parameter $M>\max \left|F^{\top} B_{\text {reg }} \delta\right|$, then

$$
\dot{V} \leq-\eta V^{\frac{1}{2}}
$$

where, $\eta=2\left(M-\max \left|F^{\top} B_{\mathrm{reg}} \delta\right|\right)$.

Therefore from (15), it is clear that using the controller (13) state trajectory reaches the sliding surface in finite time.

\subsubsection{Super-twisting control}

The major problem with the control (13) is chattering that results from the discontinuous term in the control. To avoid chattering problem, one can design super-twisting control as suggested by Levant (1993, 2010). Super-twisting control is a continuous control that leads to the second order sliding motion on the chosen sliding surface in finite time and also adjust the chattering phenomenon in the real applications.

Typically, super-twisting control for the system (4) is given by,

$$
u=-\left(F^{\top} B_{\mathrm{reg}}\right)^{-1}\left(F^{\top} A_{\mathrm{reg}} Z-u_{\mathrm{STC}}\right)
$$

where $u_{\mathrm{STC}}$ is given by,

$$
\begin{aligned}
u_{\mathrm{STC}} & =-k_{1}|\sigma|^{\frac{1}{2}} \operatorname{sign}(\sigma)+v \\
\dot{v} & =-k_{2} \operatorname{sign}(\sigma) .
\end{aligned}
$$

Note that, it is assumed that time derivative of the disturbance is also bounded. Let the bound on rate of change of disturbance be $|\dot{d}(t)|<\Delta$.

To show the finite time reaching at the sliding surface, differentiate the sliding surface (7) w.r.t. to time and substitute the 
control (16),

$$
\begin{aligned}
\dot{\sigma} & =F^{\top} \dot{Z} \\
& =F^{\top}\left(A_{\mathrm{reg}} Z+B_{\mathrm{reg}}(u+d)\right) \\
& =u_{\mathrm{STC}}+F^{\top} B_{\mathrm{reg}} d(t)
\end{aligned}
$$

Substitute (17) in (18), we get,

$$
\begin{aligned}
& \dot{\sigma}=-k_{1}|\sigma|^{\frac{1}{2}} \operatorname{sign}(\sigma)+\bar{v} \\
& \dot{\bar{v}}=-k_{2} \operatorname{sign}(\sigma)+F^{\top} B_{\text {reg }} \dot{d}(t)
\end{aligned}
$$

where $\bar{v}=v+F^{\top} B_{\text {reg }} d(t)$.

The finite time stability of super-twisting algorithm (STA) as represented in (19) is well studied in the literature for instance, see stability proof of STA using Lyapunov function in (Moreno and Osorio, 2012) and via geometric approach in Behera et al. (2017). In (Davila et al., 2005) finite time stability has been proved geometrically and also suggested the choice of parameters as $k_{1}=1.5 \sqrt{\Delta_{1}}$ and $k_{2}=1.1 \Delta_{1}$, where $\Delta_{1}=$ $\max \left|F^{\top} B_{\text {reg }} \dot{d}(t)\right|$.

\section{Application to inverted pendulum system}

In this section, we discuss the controller design for the ECP Model 505 inverted pendulum system to show the effectiveness of the proposed method. ECP Model 505 inverted pendulum system is different from the cart mass pendulum system. The representative picture of ECP Model 505 inverted pendulum setup (ECP, 2004) is shown in the Fig.1.

The setup consists of a pendulum rod which supports the sliding balance rod. The sliding rod is driven via a belt and pulley which in turn is driven by a drive shaft connected to a $\mathrm{dc}$ servo motor below the pendulum rod. In this setup balance and control of the pendulum rod position can be achieved by steering horizontal sliding rod in the presence of gravity. So in this model tracking of pendulum rod angle is possible, which is a unique feature of this model. The inverted pendulum model is an unstable and non-minimum phase system.

In this application, an objective is to track the pendulum angle to the reference signal in the presence of disturbance. To

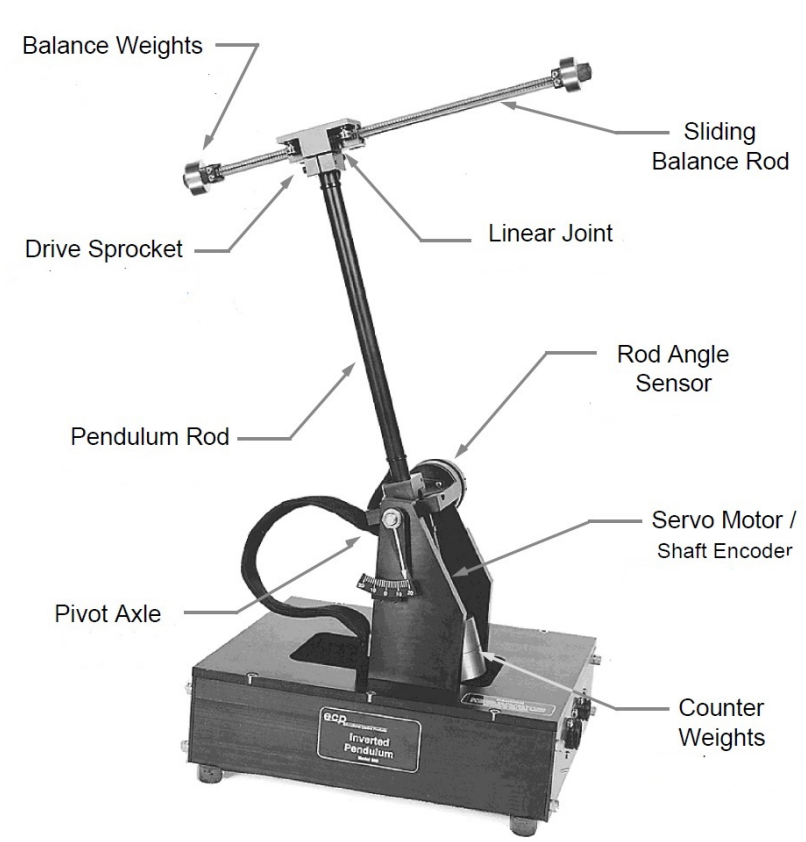

Figure 1: ECP Model 505 Inverted Pendulum.

design a controller, we need linearized model of the inverted pendulum system.

\subsection{Inverted pendulum model}

Nonlinear dynamics of the inverted pendulum system is given by,

$$
\begin{aligned}
& m_{1} \ddot{x}+m_{1} l_{o} \ddot{\theta}-m_{1} x \dot{\theta}^{2}-m_{1} g \sin \theta=F(t) \\
& m_{1} l_{o} \ddot{x}+J_{o} \ddot{\theta}+2 m_{1} x \dot{x} \dot{\theta}-\left(m_{1} l_{0}+m_{2} l_{c}\right) g \sin \theta-m_{1} g x \cos \theta=0
\end{aligned}
$$

Linearize the system about $x=0$ and $\theta=0$, we get,

$$
\begin{aligned}
& m_{1} \ddot{x}+m_{1} l_{o} \ddot{\theta}-m_{1} g \theta=F(t) \\
& m_{1} l_{o} \ddot{x}+J_{o} \ddot{\theta}-\left(m_{1} l_{0}+m_{2} l_{c}\right) g \theta-m_{1} g x=0
\end{aligned}
$$

Define, the output $y:=\theta$, input $u=F(t)$ and the state variables,

$$
X:=\left[\begin{array}{cccc}
\theta & \dot{\theta} & x & \dot{x}
\end{array}\right]^{\top}
$$

Therefore, the system (20) can be represented in state space as,

$$
\begin{aligned}
\dot{X} & =A X+B u \\
y & =C X
\end{aligned}
$$


Table 1: ECP 505 Model parameters.

\begin{tabular}{cc}
\hline Symbol & Values \\
\hline$m_{1}$ & $0.213 \mathrm{Kg}$ \\
$m_{2}$ & $1.7850 \mathrm{Kg}$ \\
$l_{c}$ & $-0.0295 \mathrm{~m}$ \\
$l_{o}$ & $0.330 \mathrm{~m}$ \\
$\mathrm{~g}$ & $9.81 \mathrm{~m} / \mathrm{s}^{2}$ \\
$J_{o}$ & $0.0595 \mathrm{Kg} \mathrm{m}^{2}$ \\
\hline
\end{tabular}

where,

$$
\begin{aligned}
A & =\left[\begin{array}{cccc}
0 & 1 & 0 & 0 \\
\frac{m_{2} l_{c} g}{J^{*}} & 0 & \frac{m_{1} g}{J^{*}} & 0 \\
0 & 0 & 0 & 1 \\
\frac{\left(J^{*}-m_{2} l_{o} l_{c} g\right.}{J^{*}} & 0 & -\frac{m_{1} l_{o} g}{J^{*}} & 0
\end{array}\right], B=\left[\begin{array}{c}
0 \\
-\frac{l_{0}}{J^{*}} \\
0 \\
\frac{J_{o}}{m_{1} J^{*}}
\end{array}\right] \\
C & =\left[\begin{array}{llll}
1 & 0 & 0 & 0
\end{array}\right]
\end{aligned}
$$

and $J^{*}=J_{o}-m_{1} l_{o}^{2}$.

For the experiment we have considered donut weight on the both side of sliding road and also two balance weight below the pendulum road. After substituting parameter values from Table 1 , we get

$$
\begin{aligned}
A & =\left[\begin{array}{cccc}
0 & 1 & 0 & 0 \\
-14.1916 & 0 & 57.4827 & 0 \\
0 & 0 & 0 & 1 \\
14.4932 & 0 & -18.9693 & 0
\end{array}\right], B=\left[\begin{array}{c}
0 \\
-9.0783 \\
0 \\
7.6907
\end{array}\right] \\
C & =\left[\begin{array}{llll}
1 & 0 & 0 & 0
\end{array}\right] .
\end{aligned}
$$

\subsection{Controller Design}

In order to design a sliding mode control, first we transform the system (21) into regular form using transformation,

$$
Z:=\left[\begin{array}{c}
Z_{1} \\
Z_{2}
\end{array}\right]:=T X, \text { with } T=\left[\begin{array}{cccc}
1 & 0 & 0 & 0 \\
0 & 1 & 0 & 1.1805 \\
0 & 0 & 1 & 0 \\
0 & 0 & 0 & 0.1300
\end{array}\right]
$$

and

$$
Z_{1}:=\left[\begin{array}{c}
z_{1} \\
z_{2} \\
z_{2}
\end{array}\right], Z_{2}:=z_{4}
$$

The transformed system in regular form is obtained as,

$$
\begin{aligned}
& \dot{Z}=A_{\text {reg }} Z+B_{\text {reg }} u, \\
& y=C Z
\end{aligned}
$$

where

$$
A_{\text {reg }}=\left[\begin{array}{ll}
A_{11} & A_{12} \\
A_{21} & A_{22}
\end{array}\right], B_{\text {reg }}=\left[\begin{array}{l}
B_{1} \\
B_{2}
\end{array}\right], C=\left[\begin{array}{ll}
C_{1} & C_{2}
\end{array}\right] .
$$

and

$$
\begin{aligned}
A_{11} & =\left[\begin{array}{ccc}
0 & 1 & 0 \\
2.9181 & 0 & 35.0889 \\
0 & 0 & 0
\end{array}\right], A_{12}=\left[\begin{array}{c}
-9.0783 \\
0 \\
7.6900
\end{array}\right] \\
A_{21} & =\left[\begin{array}{ccc}
1.8847 & 0 & -2.4667
\end{array}\right], A_{22}=0 \\
B_{1} & =\mathbf{0}_{3 \times 1}, B_{2}=1, C_{1}=\left[\begin{array}{lll}
1 & 0 & 0
\end{array}\right], \text { and } C_{2}=0 .
\end{aligned}
$$

\subsubsection{Sliding surface design}

For the sliding surface $\sigma=F_{1} Z_{1}+Z_{2}-G r$, the reduced order system dynamics during sliding motion is given by,

$$
\dot{Z}_{1}=\left(A_{11}-A_{12} F_{1}\right) Z_{1}+A_{12} G r .
$$

The design requirement for sliding motion (22) is that the overshoot is less than $10 \%$ and settling time is less than 2 second. These specifications can be satisfied by the surface parameter

$$
F_{1}=\left[\begin{array}{lll}
0.9997 & 0.3481 & 3.2895
\end{array}\right]
$$

Scalar gain $G$ can be computed from the (8), after calculation we obtained it as $G=0.7262$.

\subsubsection{Super-twisting control and first order SMC}

The first order controller (13) or super-twisting (16) can be used to reach the sliding surface in finite time. To demonstrate the robustness property, we introduce disturbance $d=\sin t$. Clearly, $\delta=\max |d|=1$, so $\left|F^{\top} B_{\text {reg }} \delta\right|=1$. Therefore, we 
chose $M=3$, which is greater than $\left|F^{\top} B_{\text {reg }} \delta\right|$. Thus, first order sliding mode controller (13) for the system in transformed coordinate is given by,

$$
\begin{aligned}
u=- & 2.9005 z_{1}-0.9997 z_{2}-9.7477 z_{3} \\
& -34.3718 z 4-3 \operatorname{sign}(\sigma)
\end{aligned}
$$

Note that, $\dot{d}=\cos t$, so $\left|F^{\top} B_{r e g} \dot{d}\right|=1$. Therefore, we chose $k_{1}=$ 3.97 and $k_{2}=7.70$. Thus, super-twisting (16) that enforces second order sliding motion on the designed surface in finite time is given by

$$
\begin{aligned}
u=- & -9005 z_{1}-0.9997 z_{2}-9.7477 z_{3}-34.3718 z 4 \\
& -3.97|\sigma|^{\frac{1}{2}} \operatorname{sign}(\sigma)+\int_{0}^{t} 7.7 \operatorname{sign}(\sigma)
\end{aligned}
$$

\section{Simulation and experiments results}

The performance of the proposed method is tested on ECP Model 505 inverted pendulum setup with the disturbance of the form $d(t)=\sin t$, in the simulation and experiment both. The controller is implemented through MATLAB Simulink RTWT (real time windows target) with sampling time $0.001 \mathrm{sec}$. The control objective was to track the pendulum angle to the desired constant value in the presence of the disturbance.

The obtained results in simulation and experiment both are quite satisfactory in the presence of disturbance. We have implemented both first order SMC and SOSM controller on the ECP inverted pendulum setup in simulation and experiment. The results are almost matching to the desired performance specifications of overshoot less than $10 \%$ and settling-time less than 2 seconds.

In the experiment, initially we have arranged manually sliding rod such that the inverted pendulum is stable at an unstable equilibrium point. From this initial condition, we have given the set point of 10 degree, after ten second pendulum was brought back to the original position, after twenty second negative 10 degree set point was given. The tracking performance of the pendulum angle $\theta$ in simulation and experiment using first order SMC is shown in the Fig. 2(a) and Fig. 2(b). The controller perfectly tracks the output in the presence of disturbance in simulation, while in experiment nearly $8 \%$ steady state error is observed. Due to the discontinuous nature of control in first order SMC, sliding rod which is connected with dc servo motor exhibits vibration which affects the pendulum angle. As we know that chattering is a practical phenomenon, which is observed in the zoom view of the Fig. 2(b). The required control effort to maintain the desired performance in simulation and experiment is shown in Fig. 2(c) and (d) respectively. Finite time reaching to the sliding surface is depicted in Fig. 2(e) and (f).

As compare to the first order SMC, STC generates continuous nature of control and obtains the desired performance without chattering. The performance of the pendulum angle in simulation and experiment using STC is depicted in Fig. 3(a) and (b) respectively. In the zoom view of the pendulum angle, it is evident that the chattering is not exhibited with STC. STC perfectly tracks the output in the presence of disturbance in simulation. However, in the experiment, steady state error observed is less than $2 \%$, which can be seen in the inset zoom window in Fig. 3(b). Note that, in simulation such error has not been observed as a constant $G$ as in (8) is computed for the identified model of the system. However, due to mismatch in the model and plant, there always exists small amount of steady state error in the practical situation even for the appropriate control efforts.

The time evolution of STC input is shown in Fig. 3(c) and (d) respectively for simulation and experiment. Finite time reaching to the sliding surface is depicted in Fig. 3(e) and (f). As mentioned earlier that STC is continuous in nature, which minimize the chattering effect on the pendulum position. Hence, overall performance using the proposed control technique is quite satisfactory, which can be observed in the results.

\section{Conclusion}

In this paper, output tracking of constant reference input for non-minimum phase system using second order sliding mode approach is proposed. To achieve tracking a new sliding surface is proposed that and to ensure sliding motion super-twisting control (STC) is used, which also adjusts the chattering. The 
proposed method is validated in simulation and experimentally both on ECP Model 505 inverted pendulum system, which is unstable, and non-minimum phase system. The obtained results are more promising using proposed control technique.

\section{References}

Bandyopadhyay, B., Fulwani, D., 2009. A robust tracking controller for uncertain mimo plant using non-linear sliding surface. In: Industrial Technology, 2009. ICIT 2009. IEEE International Conference on. IEEE, pp. 1-6.

Bandyopadhyay, B., Patil, M., 2015. Sliding mode control with the reducedorder switching function: an scb approach. International Journal of Control 88 (5), 1089-1101.

Bartolini, G., Ferrara, A., Usai, E., 1998. Chattering avoidance by second-order sliding mode control. IEEE transactions on Automatic Control 43 (2), 241246.

Behera, A. K., Chalanga, A., Bandyopadhyay, B., 2017. A new geometric proof of super-twisting control with actuator saturation. Automatica.

Boiko, I., 2008. Discontinuous control systems: frequency-domain analysis and design. Springer Science \& Business Media.

Byrnes, C., Isidori, A., 2000. Output regulation for nonlinear systems: an overview. International journal of robust and nonlinear control 10 (5), 323337.

Chalanga, A., Kamal, S., Bandyopadhyay, B., 2015. A new algorithm for continuous sliding mode control with implementation to industrial emulator setup. IEEE/ASME Transactions on Mechatronics 20 (5), 2194-2204.

Chalanga, A., Plestan, F., 2017. Finite time stabilization of an uncertain chain of integrators by integral sliding mode approach. IFAC-PapersOnLine 50 (1), 9613-9618.

Chen, B. M., Lee, T. H., Peng, K., Venkataramanan, V., 2003. Composite nonlinear feedback control for linear systems with input saturation: Theory and an application. IEEE Transactions on automatic control 48 (3), 427-439.

Davila, J., Fridman, L., Levant, A., 2005. Second-order sliding-mode observer for mechanical systems. IEEE transactions on automatic control 50 (11), 1785-1789.

ECP, 2004. User manual of ecp model 505 inverted pendulum. Educational Control Products, California, USA.

Edwards, C., Spurgeon, S., 1996. Robust output tracking using a sliding-mode controller/observer scheme. International Journal of Control 64 (5), 967983.

Edwards, C., Spurgeon, S., 1998. Sliding mode control. Bristol, PA.

Francis, B. A., Wonham, W. M., 1976. The internal model principle of control theory. Automatica 2, 457-465.

Franklin, G. F., Powell, J. D., Emami-Naeini, A., Powell, J. D., 1994. Feedback control of dynamic systems. Vol. 3. Addison-Wesley Reading, MA.

Hung, J. Y., Gao, W., Hung, J. C., 1993. Variable structure control: A survey. IEEE transactions on industrial electronics 40 (1), 2-22.
Isidori, A., 2013. The zero dynamics of a nonlinear system: From the origin to the latest progresses of a long successful story. European Journal of Control 19 (5), 369-378.

Isidori, A., Byrnes, C. I., 1990. Output regulation of nonlinear systems. IEEE Trans. on Automatic Control 35 (2), 131-140.

Kamal, S., Chalanga, A., Moreno, J. A., Fridman, L., Bandyopadhyay, B., June 2014. Higher order super-twisting algorithm. In: 2014 13th International Workshop on Variable Structure Systems (VSS). pp. 1-5.

Khimani, D., Rokade, R., 2017. Implementation of sliding mode control on magnetic levitation system. In: Advances in Computing, Communication and Control (ICAC3), 2017 International Conference on. IEEE, pp. 1-5.

Levant, A., 1993. Sliding order and sliding accuracy in sliding mode control. International journal of control 58 (6), 1247-1263.

Levant, A., 1998. Robust exact differentiation via sliding mode technique. automatica 34 (3), 379-384.

Levant, A., 2003. Higher-order sliding modes, differentiation and outputfeedback control. International journal of Control 76 (9-10), 924-941.

Levant, A., 2010. Chattering analysis. IEEE Transactions on Automatic Control 55 (6), 1380-1389.

Lukyanov, A. G., Utkin, V. I., 1981. Methods of reducing equations for dynamic systems to a regular form. Automation and Remote Control 42 (4), 413-420.

Moreno, J. A., Osorio, M., 2012. Strict lyapunov functions for the supertwisting algorithm. IEEE transactions on automatic control 57 (4), 10351040.

Patil, M., Bandyopadhyay, B., 2013a. Discrete-time sliding mode tracking control for nmp systems using reduced order switching function. IFAC Proceedings Volumes 46 (32), 469-474.

Patil, M., Bandyopadhyay, B., Feb. 2013b. High performance sliding mode tracking control for nonminimum phase systems. In: $5^{\text {th }}$ Symposium on System Structure and Control, Part of 2013 IFAC Joint Conference SSSC. Grnoble, France, pp. 96-101.

Patil, M., Bandyopadhyay, B., Chalanga, A., Arya, H., 2018. Output tracking of non-minimum phase systems via reduced order sliding mode design. IEEE/ASME Transactions on Mechatronics.

Polyakov, A., Poznyak, A., 2009. Reaching time estimation for super-twisting second order sliding mode controller via lyapunov function designing. IEEE Transactions on Automatic Control 54 (8), 1951-1955.

Shtessel, Y., Edwards, C., Fridman, L., Levant, A., 2014. Sliding mode control and observation. Vol. 10. Springer.

Utkin, V., Guldner, J., Shi, J., 2009. Sliding mode control in electro-mechanical systems. Vol. 34. CRC press.

Utkin, V. I., 1993. Sliding mode control design principles and applications to electric drives. IEEE transactions on industrial electronics 40 (1), 23-36.

Utkin, V. I., Chang, H.-C., 2002. Sliding mode control on electro-mechanical systems. Mathematical problems in Engineering 8 (4-5), 451-473.

Yan, Z., Jin, C., Utkin, V., 2000. Sensorless sliding-mode control of induction motors. IEEE Transactions on Industrial Electronics 47 (6), 1286-1297. 


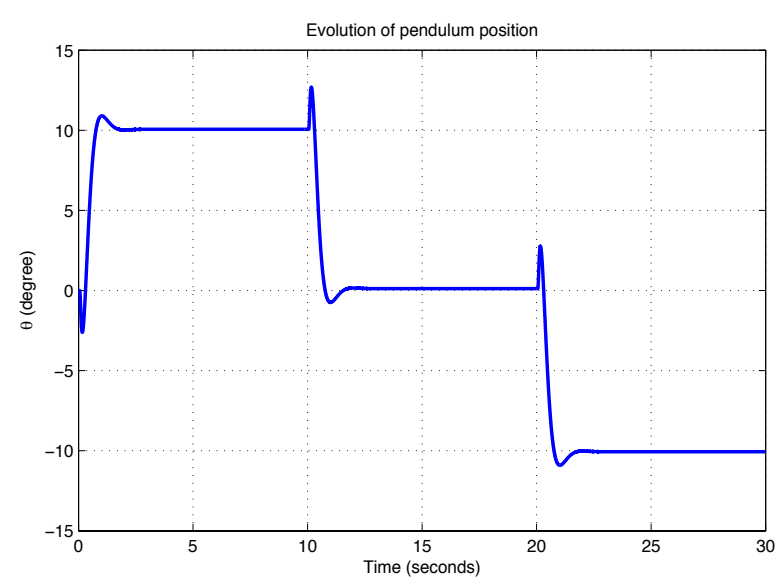

(a)

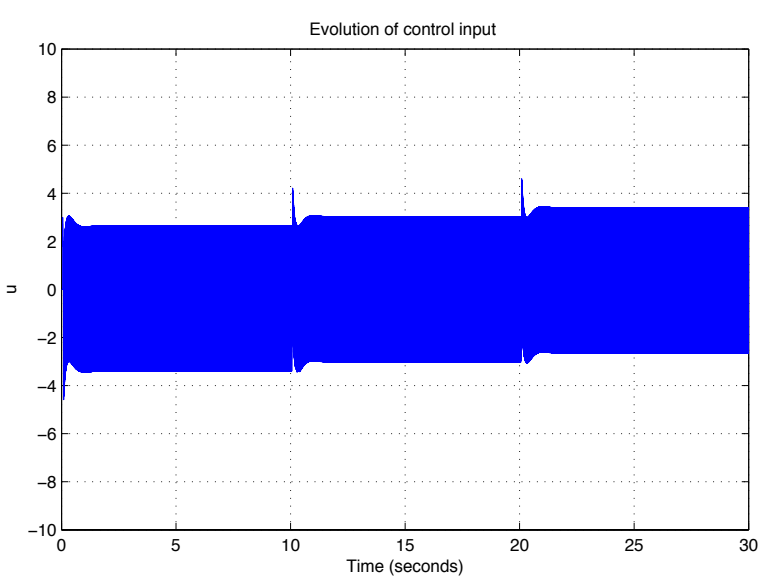

(c)

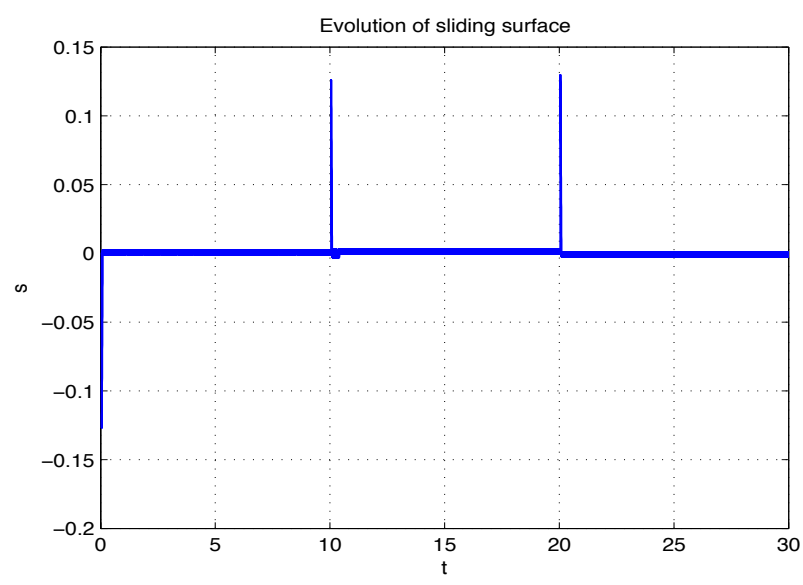

(e)

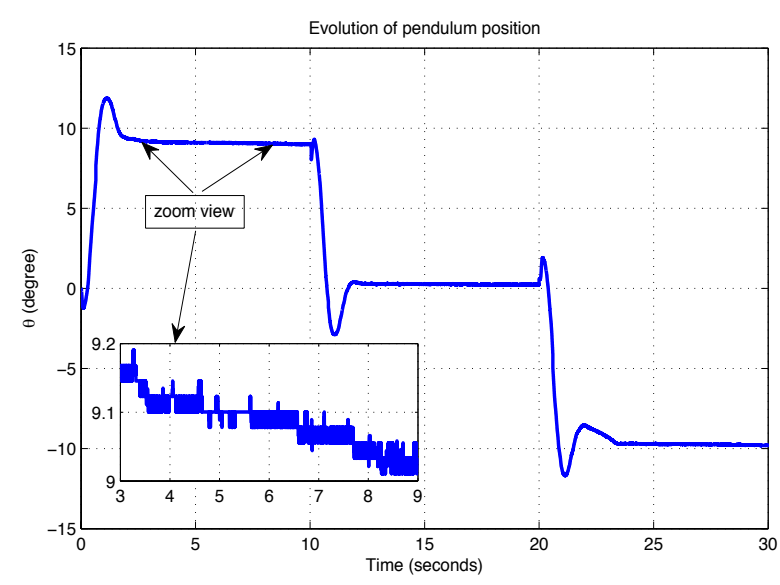

(b)

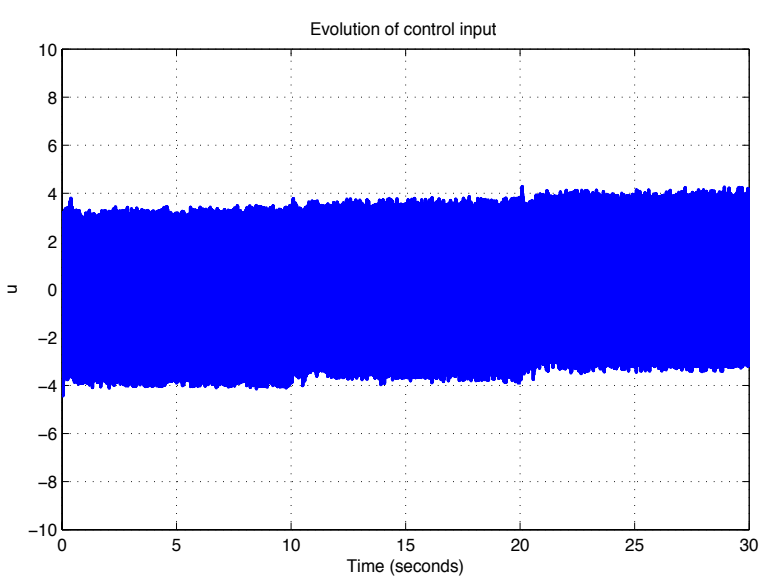

(d)

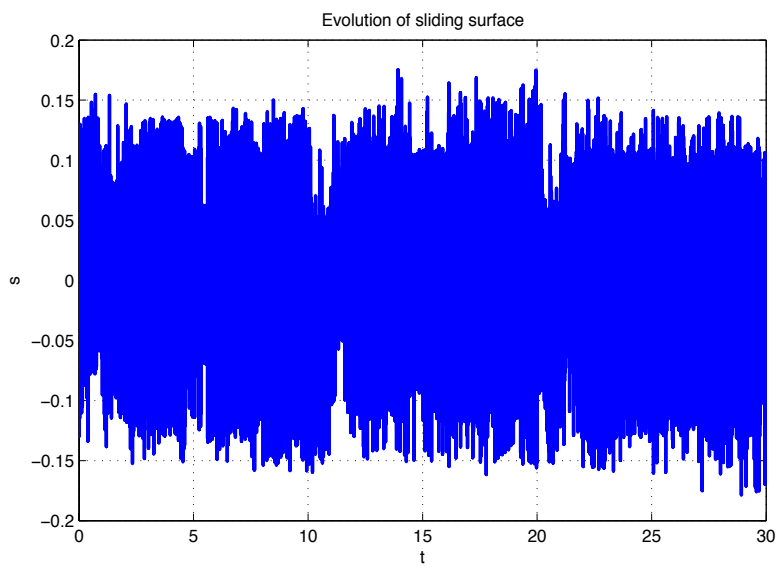

(f)

Figure 2: Simulation and Experimental Results: ECP Model 505 Inverted Pendulum with First Order Sliding Mode Control 


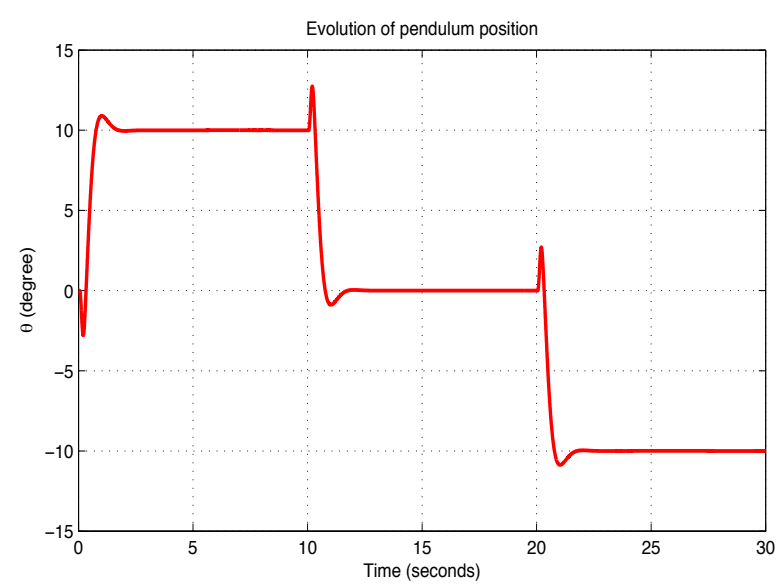

(a)

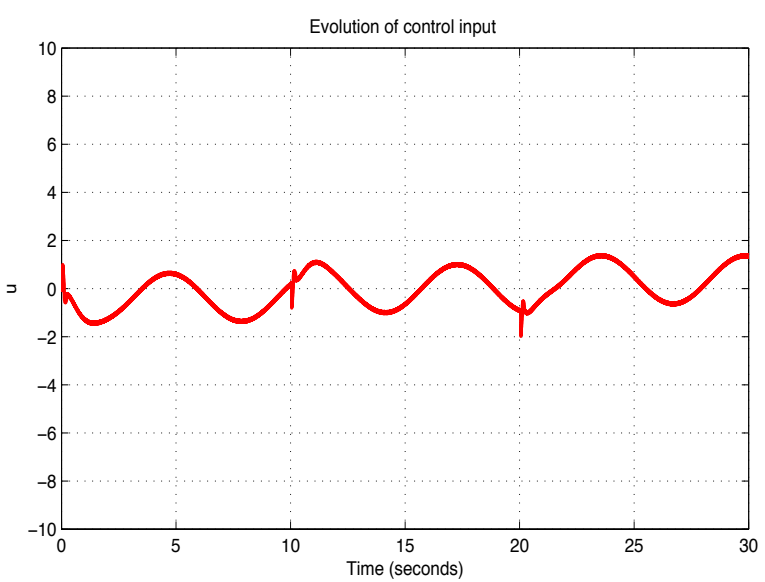

(c)

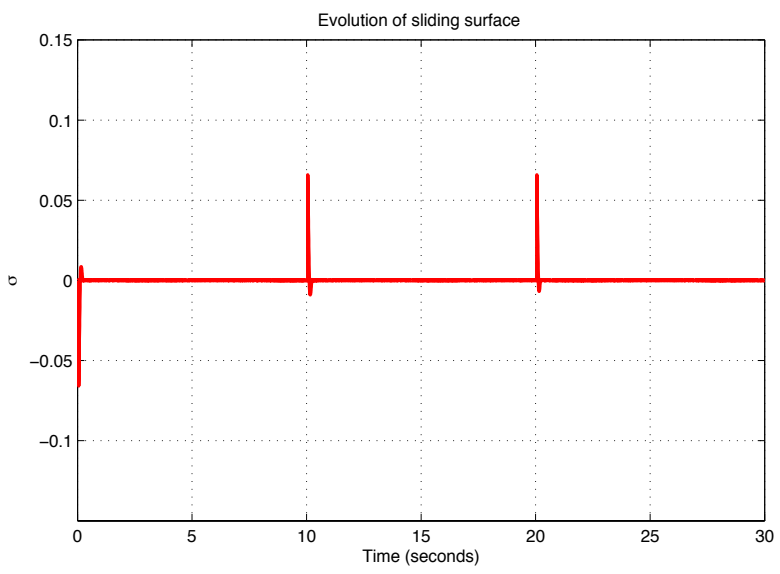

(e)

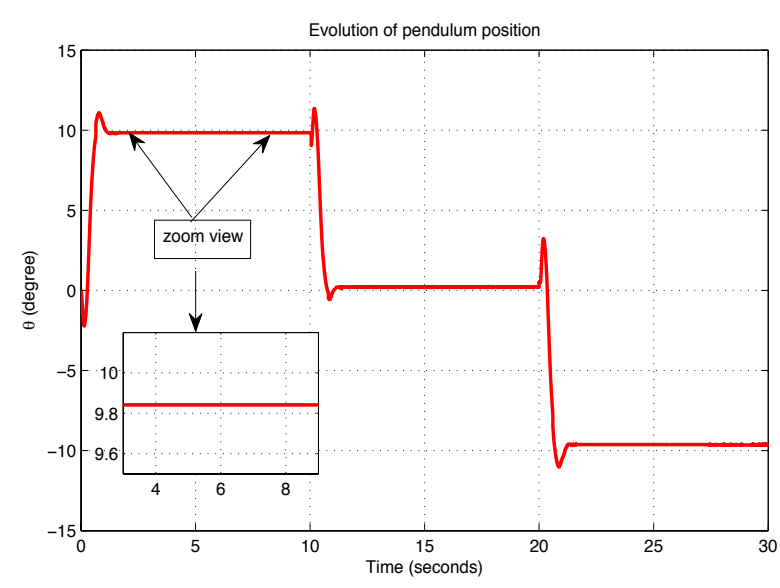

(b)

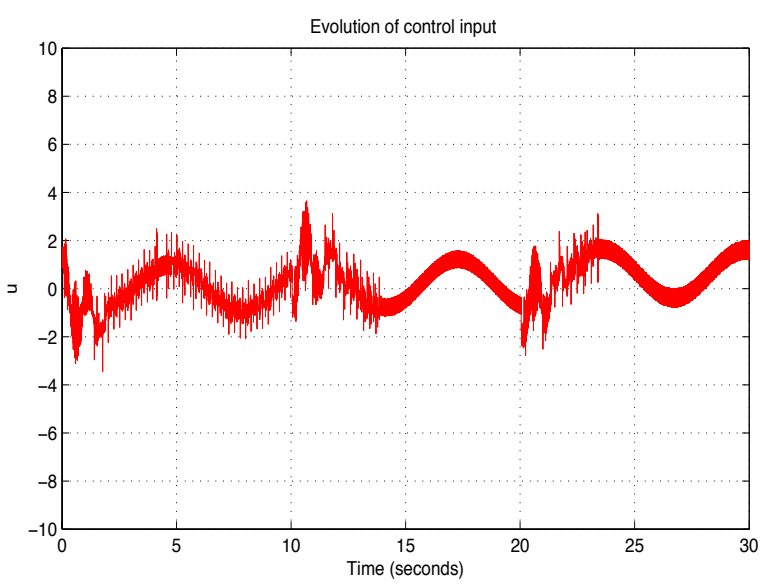

(d)

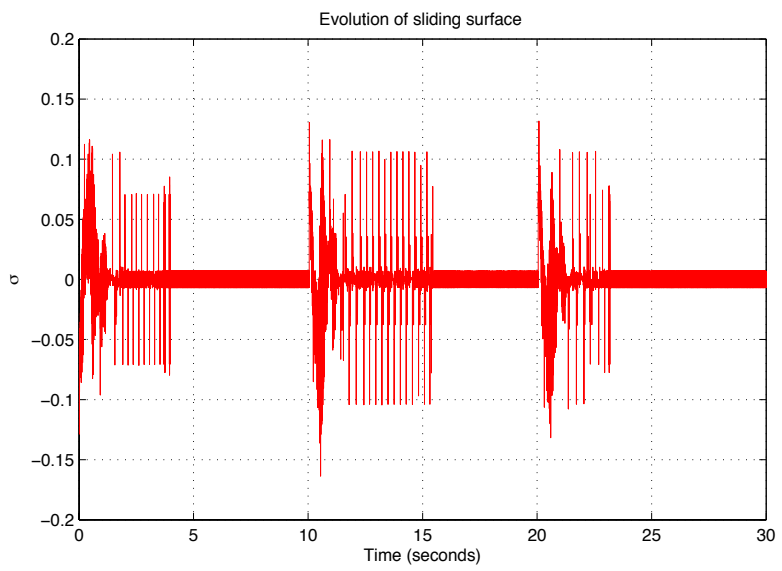

(f)

Figure 3: Simulation and Experimental Results: ECP Model 505 Inverted Pendulum with Second Order Sliding Mode Control 